\title{
Desarrollo de capacidades para el desarrollo local en cambio de época
}

\author{
Capacitação para o desenvolvimento local em tempos de mudança \\ Development of capacities for the local development on the other hand of time
}

Renforcement des capacitespour le developpement local en periode de changement

\author{
Alejandro Emilio Ramos Rodríguez* \\ (alerr@unah.edu.cu ,cedardos@infomed.sld.cu) \\ Darais Báez Fernández* \\ (daraisbf@unah.edu.cu) \\ Elaine Artigas Pérez* \\ (elaine_artigas@unah.edu.cu)
}

Recebido em 01/09/2014; revisado e aprovado em 24/10/2014; aceito em 22/11/2014

DOI: http://dx.doi.org/10.1590/151870122015209

\begin{abstract}
Resumen: El desarrollo de capacidades en los actores sean personas o colectivos necesita de marcos filosóficos, conceptuales, programáticosy metodológicos que orienten sus acciones de desarrollo, se requiere poner en práctica criterios, creatividad e imaginación para gestionar procesos en la actualización, transformación e innovación de los conocimientos, actitudes y prácticas en la solución de los problemas presentes en el municipio para la consecución del Desarrollo Local.
\end{abstract}

Palabras clave: Desarrollo local. Desarrollo de capacidades de base conceptual y metodológica. Generación de conocimientos.

Resumo: O desenvolvimento de competências nos atores são indivíduos ou grupos precisam ter estruturas filosóficas, conceituais, programáticas e metodológicas para orientar suas ações de desenvolvimento são necessários para implementar as normas, a criatividade e imaginação para gerir o processo de atualização, transformação e inovação de conhecimentos, atitudes e práticas em resolver os problemas do município para alcançar o Desenvolvimento Local. Palavras-chave: Desenvolvimento local. Desenvolvimento de capacidades de base conceitual e metodológica. Geração de conhecimento.

Abstract: The development of skills in the actors are individuals or groups need to have philosophical, conceptual, programmatic and methodological frameworks to guide their development actions are required to implement standards, creativity and imagination to manage the update process, transformation and innovation of knowledge, attitudes and practices in solving the problems in the municipality to achieve the Local Development.

Key words: Local development. Development of conceptual and methodological base capacities. Knowledge generation.

Résumé: Le développement des compétences dans les acteurs sont des individus ou des groupes ont besoin d'avoir des cadres philosophiques, conceptuels, programmatiques et méthodologiques pour guider leurs actions de développement doivent mettre en œuvre des normes, de la créativité et de l'imagination pour gérer le processus de mise à jour, la transformation et l'innovation des connaissances, attitudes et pratiques en matière de résolution des problèmes dans la municipalité de réaliser le développement local.

Mots-clés: Développement local. Renforcement des capacités, la base conceptuelle et méthodologique. La production de connaissances.

\section{Introducción}

Las sociedades se transforman al resolver práctica y culturalmente sus necesidades y sus conflictos; al hacerlo surgen nuevas necesidades y nuevos conflictos, al tiempo que es posible generar recursos, capacidades y creatividad para enfrentarlos. El principio filosófico del desarrollo humano sostenible se refiere a la permanente generación humana de necesidades, aspiraciones y expectativas, $\mathrm{y}$ al imperativo de innovar formas creativas de atenderlas sin perjudicar a las sociedades, a las comunidades, a las personas, ni al medio ambiente.

La dimensión humana del desarrollo es otro cambio paradigmático que abre una perspectiva completamente nueva para redefinir el acercamiento convencional al desarrollo. Desde esa nueva perspectiva, los seres humanos finalmente podrán convertirse en principal objeto y sujeto de su acción: ya no serán una abstracción económica sino una viviente realidad operativa, además de guías de los procesos de desarrollo cuyo control no ha estado en sus manos.

\footnotetext{
* Universidad Agraria de La Habana (UNAH), San Antonio de las Vegas, Cuba.
} 
Pero, ¿qué significa exactamente desarrollo de capacidades? Al crecer su popularidad, también parecen haber crecido ciertas confusiones al respecto. Para algunos, el desarrollo de la capacidad puede ser cualquier esfuerzo por enseñarle a alguien a hacer algo, o a hacerlo mejor. Para otros, puede significar crear nuevas instituciones o fortalecer las ya existentes. Hay quienes consideran que el desarrollo de capacidades tiene su centro en la educación y la capacitación; pero también hay quienes adoptan una visión de mayor alcance e incluyen mejorar el acceso, los derechos y las libertades individuales. Quizás todos tengan razón, lo que los significados atribuidos conllevan una visión desde la complejidad y es función de cada actor integrar para alcanzar el objetivo del desarrollo de las capacidades. Quizás a la Universidad corresponda la educación y la capacitación para el desarrollo de las capacidades, a otros actores les puede corresponder crear nuevas instituciones o brindar servicios.

\section{Desarrollo de capacidades para el Desar- rollo Local}

El desarrollo de capacidades desde una mirada coyuntural o también desde una visión estratégica del desarrollo apunta al fomento de una cultura democrática y de concertación. Así, por ejemplo, para el Centro de Estudios de Desarrollo Agrario y Rural (CEDAR) en este momento el desarrollo de capacidades está orientado a: Mejorar la capacidad de gestión de losgobiernos locales, instituciones, empresas y otros actores comomedio para lograr el cumplimiento óptimo de las nuevas competencias que son transferidas por el Gobierno Central en el marco de la descentralización (OJEDA, 2012).

Sin dejar de prestar atención a esta legítima necesidad coyuntural, es preferible inscribirse dentro de un horizonte mucho mayor. Todos intuimos o somos conscientes de que la inversión en capacidades es una condición fundamental para el desarrollo. Las capacidades implican generar o consolidar una voluntad firme para desplegar recursos materiales y no materiales en las personas y colectivos, a fin de hacer realidad sus propósitos.

Sin embargo, la voluntad es fundamental, más no suficiente: Las personas y los colec- tivos necesitan contar con marcos filosóficos, conceptuales, programáticosy metodológicos que orienten sus acciones de desarrollo. Pero además, se requiere poner en práctica criterios, creatividad e imaginación para gestionar procesos, entre los que se encuentra la gestión de la información, la gestión del aprendizaje, la gestión de la diversidad y la gestión de la incertidumbre, entre otros aspectos (OJEDA, 2013).

Es decir, para la consecución de Municipios Inteligentes, en este caso Nueva Paz, el objetivo fundamental del desarrollo de capacidades tiene que ver con que los actores, sean instituciones, personas y/o comunidades, se empoderen y logren real autonomía para administrar su vida y su destino.

Como manifiesta Max-Neef (1993, p. 33)

[...] es mediante la generación de autodependencia, a través del protagonismo real de las personas en los distintos espacios y ámbitos, que pueden impulsarse procesos de desarrollo sinérgicos en la satisfacción de dichas necesidades.

En la autonomía a nivel municipal, la descentralización es una aspiración en proceso de materializarse que, a pesar de tener ya una base legal, no es todavía irreversible. Debemos distinguir claramente entre esta aspiración que se comparte, y la voluntad política de los gobernantes en sus diferentes niveles, que muchas veces está bastante lejos de esta impostergable demanda histórica (MIMDES - DFID, 2008).

Es necesario tomar en cuenta diferentes aspectos, porque el desarrollo de capacidades no ocurre en una dimensión única, ni lineal. Por el contrario, cruza una serie de dimensiones en la misma medida que el comportamiento de las personas expresa una respuesta a una realidad multifactorial.

La primera lección que se desprende de lo expuesto es que no basta hacer una estratificación de los actores de la formación de capacidades de acuerdo a sectores, cargos, funciones o clases, sino que al interior de estos estratos también se presentan una diversidad de condiciones.

Por ello, un factor a considerar en la formulación y gestión del Desarrollo de Capacidades, en la experiencia del Municipio Nueva Paz tiene que ver precisamente con la gestión de la diversidad. De primera impresi- 
ón, el reconocimiento de esta situación podría desanimarnos un poco, dado que supondría generar respuestas para cada realidad específica y el número resultante de casos podría ser inmanejable. Justamente aquí se pone a prueba la capacidad creativa para diseñar un sistema que resolviendo el tema de la diversidad, permita a la vez ser eficientes y consecuentes con los dichos propósitos.

Diferentes actores locales del municipio coinciden en que la descentralización es una apuesta estratégica para activar el proceso de desarrollo sobre la base del desarrollo de las capacidades y potencialidades del municipio. Estando clara la apuesta, entonces la palabra clave es activar. Es decir, que partiendo de reconocer que la descentralización es un proceso de largo plazo, el reto se plantea en términos de cómo hacer para que su institucionalización sea más visible y tangible para la población local del municipio Nueva Paz.

La necesidad de activar el proceso marca la orientación del desarrollo de capacidades. Se reconoce que todos son sujetos de desarrollo de capacidades, no obstante se tiene que definir estratégicamente aquellos actores centrales que permitan catalizar el proceso. Sin embargo, si no se aborda bien este aspecto se podría caer en la tentación de trabajar solo con los iluminados o los empoderados formalmente, terminando por ser excluyentes y yendo en sentido contrario a lo que se está buscando (MIMDES - DFID, 2008).

También se podría estar tentado, como casi siempre se hace, de pensar que trabajando con los seleccionados, ellos y ellas van a hacer las réplicas necesarias. La realidad dice que esto no siempre sucede así, por lo tanto se debe hacer real el asunto, o en otras ocasiones se piensa que con capacitarse a través de un taller o un curso ya se está en condiciones de transformar la realidad, se da por hecho que la percepción e interpretación de los participantes está a tono con los objetivos propuestos en la capacitación. Por más esfuerzo que se le ponga a la selección de los actores, se observa que muchas veces los factores del entorno son más confusos y algunos de los capacitados terminan siendo absorbidos por la inercia de siempre o no construyen los conocimientos necesarios para su actualización. Un excelente facilitador de un gobierno local podría verse frustrado ante una actitud autoritaria del presidente de la asamblea o jefe de la administración y terminar asimilándose al sistema por conservar su puesto o evitar momentos desagradables.

La articulación seria y comprometida de los actores a un proceso social es una condición que se debe saber identificar. Concomitante a ello, es importante tomar en cuenta su legitimidad social, pues esta permitirá inferir el grado de magnificación de la formación y desarrollo de capacidades. La firma de compromisos y los planes de incidencia de la formación son factores que coadyuvan, pero se debe estar claro en que la mejor motivación es la que sale desde adentro, más allá de incentivos o presiones.

El desarrollo humano se vincula con el fortalecimiento de determinadas capacidades relacionadas con toda la gama de cosas que una persona puede ser y hacer en su vida; en la posibilidad de que todas las personas aumenten su capacidad humana en forma plena $y$ den a esa capacidad el mejor uso en todos los terrenos, ya sea el cultural, económico, ambiental, social y político, es decir, en un fortalecimiento de capacidades (MAX-NEEF, 1993).

El Desarrollo de Capacidades es para el CEDAR (2012) “El proceso en el cual personas, organizaciones y la sociedad incrementan sus conocimientos actitudes y prácticas en los ámbitos técnico-productivos y socio-organizativos para responder a las contradicciones internas y externas y sostenerse en el tiempo".

Para el Desarrollo de Capacidades en el Municipio de Nueva Paz fue preciso tener en cuenta la necesidad de comprender por los actores, que para ello hay que cambiar paradigmas, cambios personales y organizacionales, mejora de la calidad de las prácticas y cambios de comportamientos, entre otras exigencias. Los actores del municipio debieron comprender además que, Aprender es apropiarse de conocimientos significativos del contexto de actuación de los actores, para la solución de los problemas. Desaprender, en este caso es cuando se dan cuenta de la necesidad de un cambio, de despojarse, de borrar de la mente, conocimientos, actitudes y prácticas para la solución de los problemas nuevos y actuales que no pueden ser resueltos con los métodos y técnicas tradicionales. Reaprender, es actualizar, transformar e innovar 
los conocimientos, actitudes y prácticas en la solución de los problemas presentes en el municipio, son los nuevos métodos, tecnologías, estilos que permiten la transformación de los problemas presentes, que con los conocimientos, actitudes y prácticas anteriores, es decir, tradicionales, más que transformar el problema este se acrecienta, se hace más complejo y sin solución.

Para la formación y desarrollo de capacidades en el municipio Nueva Paz se tuvo en cuenta los componentes siguientes: ¿Saber qué?, el ¿Saber por qué?, el ¿Saber para qué? y el ¿Saber cómo?

\begin{tabular}{|c|c|c|}
\hline \multicolumn{3}{|c|}{ DESARROLLO DE CAPACIDADES Y FORMACION DE COMPETENCIAS } \\
\hline Saber qué: & $\begin{array}{l}\text { DATOS - ESTADÍSTICAS } \\
\text { - HECHOS }\end{array}$ & Para conocer y controlar larealidad. \\
\hline Saber por qué: & INFORMACION & $\begin{array}{l}\text { Análisis, comprensión, interpretación de datos, } \\
\text { hechos y estadísticas de la realidad. }\end{array}$ \\
\hline \multirow{3}{*}{ Saber para qué: } & CONOCIMIENTOS & $\begin{array}{l}\text { Un estado cambiante de comprensión y } \\
\text { aceptación de las premisas (verdades) } \\
\text { constitutivas de dicha comprensión, que emerge } \\
\text { después de un esfuerzo de análisis y síntesis. }\end{array}$ \\
\hline & HABILIDADES & $\begin{array}{l}\text { Operaciones intelectuales y psicomotoras del } \\
\text { sujeto en su relación con la realidad. }\end{array}$ \\
\hline & VALORES & $\begin{array}{l}\text { Rasgos transcendentes de la personalidad } \\
\text { (sentimientos - convicciones). Son las virtudes } \\
\text { que orientan la actuación del sujeto en la realidad. }\end{array}$ \\
\hline \multirow{2}{*}{ Saber cómo: } & CAPACIDADES & $\begin{array}{l}\text { Acciones intelectuales del sujeto que lo faculta } \\
\text { para transformar la realidad: Investigar, diseñar, } \\
\text { sistematizar, desarrollar metodologías para } \\
\text { transformar y obtener cambios en la realidad. }\end{array}$ \\
\hline & COMPETENCIAS & $\begin{array}{l}\text { Potencialidades funcionales de las facultades } \\
\text { del sujeto - son las aptitudes del sujeto que le } \\
\text { permiten actuar y contextualizar para cambiar } \\
\text { en otra realidad cualitativamente mejor. }\end{array}$ \\
\hline
\end{tabular}

Las capacidades pueden según la literatura ser de dos tipos:

Las Funcionales: Son capacidades "transversales" que están asociadas a todos, a los diferentes niveles y no a un sector o tema en particular. Son las capacidades de gestión necesarias para formular, implementar y revisar políticas, estrategias, programas y proyectos.Como las capacidades funcionales se centran en "lograr que las cosas se hagan", son de importancia clave para el éxito del desarrollo de capacidades, cualquiera sea la situación subyacente.

Las Técnicas: Son las que están asociadas a áreas particulares de experticia y práctica de sectores o temas específicos tales como el cambio climático, VIH/SIDA, deporte comunitario, cultura, salud, o como tales, están estrechamente relacionadas a un sector u organización en cuestión.
Por ello a partir del esquema anterior, el CEDAR, da valor agregado al Desarrollo de Capacidades y señala, "el desarrollo de capacidades es el proceso mediante el cual los individuos, grupos, organizaciones, instituciones, comunidades y sociedades incrementan su información, conocimientos, habilidades, actitudes, valores, para realizar funciones esenciales, resolver problemas, definir y lograr objetivos y entender y responder a sus necesidades de desarrollo en un contexto y de manera sostenible" (CEDAR, 2013, p. 22).

\section{La gestión de los procesos de formación}

Este es un punto central para la elaboración de un programa de Desarrollo de Capacidades en el municipio. Se parte de una realidad específica en cada lugar, en este caso el Municipio Nueva Paz, con su propia histo- 
ria, sus propios actores, sus propios procesos políticos, sociales, económicos, ambientales y culturales y, sobre todo, con su propia base de recursos naturales. Esto hace la realidad peculiar y única. No obstante, hay marcos genéricos que se aplican como país.

En principio, son los actores locales quienes debieran formular su Programa de Desarrollo de Capacidades, porque ellos conocen mejor, qué es lo que tienen, qué es lo que quieren y cómo lo quieren hacer. Sin embargo, también es cierto que nuestro proceso de descentralización se inscribe en un marco en el que el gobierno central, el gobierno provincial y el gobierno local tienen funciones que cumplir dentro de una lógica de integración y de unidad.

Por ello, existen también planes y programas nacionales de Capacitación y Asistencia Técnica en Gestión Pública para el fortalecimiento de los Gobiernos provinciales y locales, los cuales deben contextualizarse de acuerdo a las necesidades e intereses de los territorios y municipios para la transformación de su entorno, lo que posibilita el accionar en el campo del desarrollo de capacidades locales.

Habiendo precisado los roles en el $\mathrm{Mu}-$ nicipio Nueva Paz, se pudo atender la gestión del proceso. Se partió de la problemática específica del municipio, pero además se contó con el potencial específico que hace referencia a la energía cultural presente en cada proceso, así como a los recursos materiales y las personas calificadas con que se cuenta para sacar adelante tanto la formulación del Programa de Desarrollo de Capacidades como su gestión, monitoreo y evaluación.

Es importante conocer tanto la problemática como las potencialidades. Sin embargo, para efectos de la realidad en el municipio de estudio, con carencias materiales y déficit de capacidades de los actores en su actualización, se presta más atención a las potencialidades, pues es lo que actualmente se posee y con lo que se puede contar al margen de la provisión de recursos externos, tan esquivos $y$ tan escasos (MIMDES - DFID, 2008).

Una creativa mirada a las potencialidades en el Municipio Nueva Paz ayudó a poner en valor las energías, las capacidades y las facultades con que se cuenta. Sobre estos factores se puede decidir, lo que hace más independientes y menos vulnerables a las eventualidades de los procesos externos. Por ello, una herramienta de inestimable valor en la consecución de Municipio Inteligente en Nueva Paz lo constituyó el Mapeo de Actores Sociales (POZO, 2007).

En el Municipio Nueva Paz, el aprovechamiento de las potencialidades pasó, ante todo, por identificar personas y procesos que magnificaron el accionar continúo, con la construcción de alianzas estratégicas entre gobiernos locales o provinciales con espacios socio-educativos como la Universidad y el Centro Universitario Municipal donde ya existe experiencia instalada en temas que interesa desarrollar, y llegó hasta la definición de espacios estratégicos de formación que no necesariamente coinciden con límites geográficos o políticos.

Lo importante es que los espacios identificados permitieron hacer más eficientes los procesos de formación. Pero además, significó poner en valor las experiencias de gestión, de actores y procesos muchas veces no documentados ni ordenados académicamente, pero muy ricos en lecciones y metodologías tanto para saber hacer las cosas, como para no volver a cometer los mismos errores. En este contexto la fábrica, la empresa, el sindicato, la asociación campesina, el local del médico de la familia, entre otros espacios, también cobran valor de aprendizaje. En el fondo, todo esto no tiene otro objetivo más que institucionalizar una sociedad de aprendizaje.

Cuando se hace referencia a la formación y desarrollo de capacidades, conviene precisar que se está aludiendo a toda la gama de espacios, tiempos y oportunidades de aprendizaje que ofrece la vida. Es decir, procesos de formación formal, informal y no formal. Ahora bien, independientemente de cualquier modalidad siempre se debe tener objetivos claros, hacer explícitos los resultados esperados y contar con una metodología definida.

\section{El desarrollo de capacidades para el actu- al proceso de desarrollo local}

El propósito central es avanzar hacia una cultura democrática y concertadora, tomando el actual proceso de descentralización como un medio excepcionalmente favorable 
para alcanzarla. Pero también se debe ser consciente que este es un proceso de muy largo aliento en el que tienen que ser bien dosificadas las energías individuales e institucionales para no perderse en la angustia o en la frustración y recordar siempre la frase de ese gran pensador que fue Don Fernando Ortiz "Ciencia, Conciencia y Paciencia”. Sobre todo cuando se sabe que muchas de las acciones se soportan, en mayor o menor medida, en representaciones de gobiernos locales cuya temporalidad todavía está de la mano con fragilidad institucional.

En el Municipio Nueva Paz se manejó simultáneamente dos dimensiones: Una dimensión de visión a largo plazo, como proceso, y una dimensión de visión a corto plazo, como gobierno. Además, es necesario tomar como un hecho razonablemente esperado que del conjunto de actores formado en los programas habrá quienes deserten, algunos que se desplacen y otros que se quedarán para fortalecer sus capacidades. La apuesta, sin embargo, es lograr la suficiente motivación como para que la mayoría se quede acompañando eficientemente sus procesos locales.

Se consideró, junto a lo dicho, que no se está trabajando para formar una sola y única promoción, sino que el esfuerzo se dirige a un proceso constante y permanente de actualización de nuevos actores, con lo cual se puede mitigar el riesgo que significa la alta movilización de personal.

Se necesitó entonces en Nueva Paz, para la coyuntura, resolver las necesidades de formación y desarrollo de capacidades según el grado de importancia. Para ello se dirigió por los actores a mejorar la capacidad de gestión y decisiones del gobierno local, generando apropiados cuadros gerenciales, profesionales y técnicos que den soporte y viabilidad al proceso de descentralización y promuevan el desarrollo local con equidad (OJEDA, 2007).

Desde el Municipio se busca por sus actores generar liderazgos que aseguren las bases de la competitividad en la prestación de servicios públicos locales eficientes, que se refuerce una cultura organizacional donde la creatividad, el trabajo en equipo, la calidad del servicio al usuario, el trabajo por resultados y el orgullo de pertenencia sean sus distintivos.

Sin embargo, el municipio actualiza, aprende y desarrolla capacidades para la transformación del entorno y la consecución del desarrollo local, por lo que:

- Consolida el proceso de programas sociales y logra la mejor gestión local.

- Desarrolla capacidades de gestión del gobierno local para fortalecer el proceso de descentralización.

- Consolida procesos participativos en curso en la gobernabilidad local.

- Fortalece las capacidades de liderazgo democrático de la sociedad civil para mejorar la gobernabilidad local con su participación activa en la gestión y seguimiento.

Estos hitos pueden abordarse simultáneamente, pero a la vez marcan una línea de secuencialidad. Esto quiere decir que es el énfasis lo que cambia, según las necesidades que el proceso local demanda.

A pesar de todas las investigaciones realizadas en los últimos años no hay todavía una teoría completa y coherente que haya sido adoptada por los investigadores y teóricos del campo de la educación de adultos; prueba de ello son las diversas definiciones que existen sobre este concepto; sin embargo, para avanzar en el logro del desarrollo de capacidades, en el proyecto se ha trabajado sobre el aprendizaje individual permanente y el trabajo en grupos desde la Metodología de la Educación Popular.

\section{La Educación para el Desarrollo de Capa- cidades}

El Desarrollo de Capacidades en los programas y proyectos que se desarrollan en Nueva Paz para el Desarrollo Local se nutre de diversas fuentes como: la pedagogía crítica, las corrientes constructivistas del conocimiento, que tienen su origen en la psicología de Piaget, Vigotsky, Luria o Bruner, el modelo de investigación-acción elaborado por Lewin, retomado y profundizado por Carr y Kemmis, entre otros. Siguiendo a Vigotsky quien consideraba la educación como un proceso de interacción social en el que el adulto guía al aprendiz y le introduce en la cultura, la Educación para el Desarrollo concibe la enseñanza aprendizaje como un proceso activo y participativo en el que el docente actúa como facilitador para ayudar en la construcción del conocimiento, partiendo de sus preocupaciones y centros de interés (DIEZ DEL CORRAL, 2005). 
La Educación para el Desarrollo de Capacidades en la consecución de Municipios Inteligentes como lo es Nueva Paz, se enmarca en un planteamiento educativo no tradicional, en el que se ha pasado de priorizar solo los contenidos, a considerar que la educación en actitudes, valores y habilidades es esencial y debe formar parte del proceso formativo. Por esta razón, y por su carácter crítico, la Educación para el Desarrollo de Capacidades se enfrenta a diversos problemas para su puesta en práctica en el sistema formal e informal de enseñanza. Las escuelas a menudo siguen utilizando esquemas tradicionales de aprendizaje -con una concepción del conocimiento muy compartimentalizada- que hace difícil la introducción de enfoques globales y de metodologías participativas para trabajar con grupos.

La educación para el desarrollo de capacidades, se caracteriza en Nueva Paz por su dimensión globalizadora, abordando distintos aspectos de la realidad y analizando las interrelaciones que existen desde una perspectiva local-global. En este sentido, Souza (2006) señala que es además interdisciplinaria, transdisciplinaria porque no se circunscribe a un solo sector y propone un trabajo entre los distintos sectores que trasciende a la comunidad municipal en pleno.

La educación para el desarrollo de capacidades de los actores del Municipio Nueva Paz, propugna valores solidarios, cooperativos, críticos, de participación, no se lleva a cabo mediante métodos tradicionales, jerárquicos, individualistas, de transmisión vertical de conocimiento, sobre todo si se considera que los destinatarios de la información son a su vez multiplicadores tanto de los contenidos como de los valores y actitudes que se van definiendo a lo largo del proceso formativo. Por esta razón, en la educación para el desarrollo de capacidades no se separa texto y contexto, método y contenido, es la única manera de no provocar disonancias cognitivas entre aquello que se dice, la forma en la que es trasmitido y la práctica. En definitiva, descuidar la dimensión metodológica puede conducir a una desvalorización de los contenidos, que a la postre llegan a invalidarlos, convirtiéndolos en retórica, o reduciéndolos a un valor puramente instrumental, desprovistos de su carga ética y movilizadora (DIEZ DEL CORRAL, 2005).
Las características de la metodología de grupo de formación las hace más apropiada para desarrollar una propuesta de educación para el desarrollo de capacidades de forma coherente. Sus raíces se encuentran desde el punto de vista pedagógico en algunos modelos educativos de la Escuela Nueva, de la que se trabaja en el Municipio de Nueva Paz con la propuesta de la Educación Popular (FREIRE, 1994, 2000).

Por su parte, Diez Del Corral (2005, p. 314) asegura que "El grupo como proceso, a lo largo de la actividad va a atravesar diferentes momentos, que se corresponden con diferentes fases del aprendizaje". En un primer momento la actividad se centrará en la creación del grupo y de un clima adecuado para la formación. Luego el grupo atravesará inicialmente momentos más dirigidos y de aportación de conocimientos por parte de especialistas, posteriormente el protagonismo del grupo será mayor. Por eso es muy importante concebir la actividad del grupo como un proceso en el que el recorrido es muy importante en la medida que se desarrollan capacidades para el trabajo en grupos. Una lección aprendida en el trabajo en grupo en el Municipio Nueva Paz, es que gobernantes, funcionarios, especialistas, instituciones se formaron e integraron en un grupo para el desarrollo de capacidades que posibilitó la comprensión para la transformación del entrono en la consecución de prosperidad y sostenibilidad.

En el Desarrollo de Capacidades, los actores del municipio se consideraron como vehículo y protagonistas del proceso de aprendizaje como requisito fundamental de la metodología del grupo en formación. Para ello es necesario tener en cuenta una serie de condiciones, que han de ser creadas por el educador/a y previstas en la programación y la metodología a aplicar.

Se creó el clima de grupo de formación. En este orden de ideas,se trata de confianza y empatía para poder romper barreras interpersonales y favorecer la comunicación horizontal fluida. Este clima se logró desde las primeras sesiones con la realización de actividades o dinámicas de conocimiento interpersonal. Se utilizó dinámicas que crean situaciones de juego que permiten el conocimiento interpersonal a través de otros códigos, conociendo las 
reacciones, actitudes, sentimientos y valores de las personas sin necesidad de expresar cuál es el status de cada cual en el mundo profesional o en las relaciones sociales. Este clima tiene que ser lúdico durante todo el proceso.

Se estableció un acuerdo de formación. Las expectativas y necesidades formativas de cada uno de los y las participantes son, por naturaleza, diferentes y no necesariamente coinciden con la institución, la actividad o con los educadores/as. Se explicitó las expectativas y demandas de cada cual, se contrastó, discutió y refundió en un todo que permita la creación de un interés colectivo grupal. Este paso es muy importante porque va a ser el hilo conductor de todo el proceso de aprendizaje, va a establecer unos parámetros para la evaluación final y va a permitir formular y explicitar las condiciones y requisitos que harán posible llevar a cabo el objetivo.

\section{La generación y apropiación del conoci- miento en el Desarrollo de Capacidades para el Desarrollo Local}

Con la época emergente surge un nuevo modo de generación de conocimiento (GIBBONS et al., 2004 ) con atributos que lo diferencian del modo clásico, sin que eso signifique incompatibilidad -sino complementariedad- entre los dos. Ello se tiene en cuenta por el CEDAR (2012) para la construcción de municipios inteligentes bajo la premisa de que el Programa Participativo Municipio Inteligente y los proyectos que se generan son del, con y desde el gobierno.

En este sentido,los cinco atributos del modo de generación de conocimiento en el desarrollo de capacidades son:

1. Conocimiento generado en el contexto de su aplicación e implicaciones.

2. Esfuerzo transdisciplinario.

3. Diversidad de actores y organizaciones.

4. Reflexividad y compromiso sociales.

5. Control social ampliado de la calidad y relevancia.

6.1 Conocimiento generado en el contexto de su aplicación e implicaciones

En el desarrollo de capacidades en el Municipio Nueva Paz al contrario del modo en que se generaba conocimientos con ante- rioridad sin posibilidad de tener en cuenta el contexto, pues el modo clásico de "producción" de conocimiento es "producido" dentro de organizaciones creadas exclusivamente para esta finalidad, el modo emergente aplicado en la actualidad en el municipio implica un proceso de generación de conocimiento deliberadamente comprometido con el contexto de su aplicación (dimensión práctica) e implicaciones (dimensión ética). Por eso, su práctica ocurre en el contexto, y bajo su influencia directa. Un modelo de investigación, desarrollo e innovación centrado en el entorno del municipio es el cambio más relevante para cualquier Universidad u organización de ciencia y tecnología motivada a practicar la actividad científica y tecnológica bajo la lógica del modo de generación del conocimiento para la contribución al Desarrollo Local.

Por esta razón, las acciones de investigación en la construcción de municipios inteligentes dan un giro de 180 grados para alcanzar su visión y objetivos ante métodos y prácticas muy arraigadas en todos los actores sociales que interactúan a nivel de municipio. Es importante tener en cuenta que estas posiciones que se adoptan y formadas en los actores por el paradigma clásico se desarrollan con las mejores de las intenciones, sin percibir estos actores sociales, personas e instituciones, que el grado de coherencia interna para el desarrollo de las acciones que se proponen ya no están en correspondencia con el contexto hacia donde se dirigen. En ocasiones a nivel de municipio se piensa que es algo fácil de solucionar, sin embargo, en la interacción en diferentes municipios se percibe la alta complejidad para alcanzar las transformaciones y el cambio, pues a pesar de la voluntad y del reconocimiento de la necesidad de cambio, aún prevalecen los viejos métodos y prácticas arraigadas durante la vida cotidiana.

Por su parte, el Modelo del Desarrollo Local Municipio Inteligente en Nueva Paz, en la metodología para su construcción, entre las diferentes herramientas que utiliza está el "mapeo de actores" que descansa sobre el supuesto de que la realidad social se puede ver como si estuviera conformada por relaciones sociales donde participan diversos actores sociales. Tal como lo plantea Martín Gutiérrez el abordaje de redes sociales se caracteriza por considerar que, se puede pensar 
a la sociedad en términos de estructuras las cuales se manifiestan por diferentes formas de relaciones entre actores sociales (sean estos actores, grupos, organizaciones, clases o individuos). Los conjuntos de vínculos o de relaciones sociales forman redes y según sea la posición que los distintos actores ocupan en dichas redes, van a definir sus valores, creencias y comportamientos (POZO, 2007).

Es una herramienta fundamental para la gobernabilidad que permite el análisis de los intereses de los diferentes actores implicados con una activa participación en los procesos de desarrollo en el territorio, para socializar, construir, generar, utilizar y compartir dicho conocimiento en favor del desarrollo municipal sostenible.

También será necesario crear la capacidad para reconfigurar conocimiento y experiencias. Mientras el modo clásico fue creado principalmente para generar conocimiento nuevo, universalizarlo y transferirlo, el foco principal en este caso es dirigido a la reconfiguración de conocimiento y experiencias ya existentes para los diferentes contextos que los demandan. Cuando el conocimiento y las experiencias existentes no son suficientes o no son relevantes para llenar las necesidades de un determinado contexto, el esfuerzo incluye la generación de conocimiento nuevo; pero teniendo como referencia el propio contexto (GIBBONS et al., 1994; DEMO, 1997; ENGEL; SALOMÓN, 1997; SOUZA, 2001; OJEDA, 2012).En este caso, en los municipios que se transforman en municipios inteligentes, el CEDAR y la Universidad Agraria de La Habana facilitan, educan, construyen, forman y desarrollan capacidades en los actores de la comunidad municipal pues ellos son los que conocen y comprenden como transformar sus comunidades y municipio.

Las implicaciones para la gestión de la investigación y extensión generados por el CEDAR exige de condiciones necesarias entre las que incluyen: proyectos centrados en el entorno y orientados para resolver problemas complejos, democratización del acceso a la información, conocimiento generado con la participación de los que lo necesitan, desempeño de los proyectos dependiente de la dinámica del contexto de su aplicación e implicaciones, incidencia de la diversidad de intereses externos, reconfiguración de conocimiento y experiencias existentes, incertidumbre e inestabilidad como premisas para la gestión y planificación, flexibilidad en la formulación y gestión de proyectos.

\subsection{Esfuerzo transdisciplinario}

En el municipio de Nueva Paz como en cualquier contexto, la realidad es compleja; no pudiendo ser interpretada y transformada a partir apenas del conocimiento disciplinario (GIBBONS et al., 1994; ENGEL, 1997; SOUZA, $2008,2010)$. Una vez que el modelo emerge para manejar problemas y desafíos complejos, este será un esfuerzo que va más allá de las disciplinas disponibles, al contrario del modo clásico que se basa en el aporte disciplinario.

Este tipo de esfuerzo comprometido con la complejidad del contexto de la aplicación e implicaciones del conocimiento a generar va a requerir la participación adicional en el municipio de otra categoría de expertos: aquellos talentos que aún no siendo formalmente capacitados poseen conocimiento tácito relevante sobre los problemas y desafíos complejos y sus respectivos contextos, lo que demandará el desarrollo de capacidades en los actores locales. Todo especialista es un experto, pero no todo experto tiene que ser un especialista formalmente capacitado. Involucrar talentos locales, externos a las disciplinas, es crítico para generar conocimiento comprometido con el contexto de su aplicación e implicaciones. Eso implica crear la capacidad para construir y gerenciar equipos que incluyan expertos no reconocidos entre los actores tradicionalmente asociados al modo clásico de generación de conocimiento.

Para la gestión de la innovación en el municipio, la práctica de la transdisciplinariedad implica, por ejemplo, seleccionar problemas complejos para la investigación y, después, inspirados en éstos, derivar problemas sencillos de investigación, construir marcos conceptuales para los equipos de los proyectos, gestión de proyectos transdisciplinarios, gestión de procesos participativos, actuación de los equipos bajo el enfoque sistémico, y la participación de expertos sin especialización formal; todo ello implica cambio en la forma de actuar y pensar de los actores, lo que exige el desarrollo de capacidades funcionales y técnicas. Al desarrollar capacidades en los 
actores en la manera de pensar y accionar es importante construir los conceptos, asumir la base metodológica y conceptual para la consecución del desarrollo local y las herramientas que facilitarán la consecución de Municipio Inteligente para el Desarrollo Local.

\subsection{Diversidad de actores y organizaciones}

En el municipio de Nueva Paz, objeto de investigación, y en este caso contexto de la aplicación e implicaciones del conocimiento, hay siempre la presencia de muchos actores y organizaciones interesados en este tipo de esfuerzo. Será imposible generar conocimiento socialmente relevante sin la participación de los actores que lo necesitan o que serán impactados por su uso. En ese punto, la participación se presenta desde un enfoque metodológico según Rebellato (2004), como una herramienta importante en el ámbito comunitario. Por ello, uno de los puntos clave para la participación ciudadana a nivel local, lo constituye el fomentar el sentido de pertenencia como "agente" activo que puede aportar de modo puntual, con acciones concretas, con cronogramas pautados, al abordaje de la realidad problemática a nivel local.

Todo ello se convierte entonces en el primer desafío a enfrentar por los diversos actores sociales, a la hora de construir y generar conocimientos en las dimensiones del desarrollo local: el sentirse parte en el abordaje del problema, el saberse valioso por el aporte que se puede brindar, desde la perspectiva personal, pero que además, si eso se realiza en conjunto con otros actores locales, en un diálogo abierto, franco y de respeto, se puede potenciar ese aporte, enriqueciéndose con las propuestas de otros, a través de la negociación y la concertación (ARTIGAS, 2014).

Eso implica gestión de proyectos interinstitucionales y comunitarios, formación de equipos interinstitucionales ad hoc, negociación interinstitucional y comunitaria permanente, relaciones y comunicaciones interinstitucionales permanentes, gestión permanente de conflictos y resistencias, gestión de estrategias de cofinanciación de proyectos, la actuación en red, gestión de procesos participativos. Habrá también la necesidad de actuar bajo ciertos principios orientadores previamente negociados para servir de referencia para facilitar la colaboración interinstitucional, como por ejemplo: infraestructura compartida, intercambio de talentos, autoridad del argumento (no el argumento de la autoridad) y valorización de los talentos locales.

Es condición en los proyectos de desarrollo e investigación que se desarrollan por el CEDAR en la consecución de municipios inteligentes por un desarrollo local, la generación y construcción de conocimientos de forma contextual y con interacción social a pesar de que en el presente, el modo clásico, tradicional, el positivismo ya en decadencia, se encuentra bien arraigado en la forma de actuación de los actores sociales, aunque todos sabemos que estamos abocados $i a$ cambiar! Para ello, es necesario e imprescindible el desarrollo de capacidades de manera sistemática y permanente. En consecuencia, el desarrollo de capacidades no es posible con talleres, cursos, diplomados en ocasiones sustentados por métodos tradicionales o conocimientos desactualizados, porque aún el facilitador no ha alcanzado estas capacidades y pretende transformar el entorno con lo pasado.

En el desarrollo de capacidades, el conocimiento se construye y valida desde la interacción entre los grupos sociales (interacción social), conocimientos locales y conocimientos científicos. Enfatiza el contexto de descubrimiento (conocimiento de la realidad, generación de teoría), más que el de justificación (comprobación). Conocimiento Universal - prueba de la teoría. Conocimiento contextual - Generación de teoría. Comprender para transformar (RAMOS et al., 2013).

Si bien, en el municipio Nueva Paz, este modelo también plantea un cambio de actitudes, este no debe estar asociado a la adopción de nuevas tecnologías ni al condicionamiento mecánico de conductas. El cambio fundamental aquí consiste en el paso a un hombre - mujer acrítico a un hombre mujer crítico; en ese proceso de un hombre desde los condicionamientos que lo han hecho pasivo, conformista, fatalista, hasta la voluntad de asumir su destino humano; desde las tendencias individualistas y egoístas hasta la apertura a los valores solidarios y comunitarios. Se trata de no seguir caminos para la consecución del desarrollo local, se trata de 
desarrollar capacidades para construir caminos que permitan el desarrollo local.

\subsection{Reflexividad y compromiso sociales}

Bajo la lógica del modo emergente de generación de conocimiento en el desarrollo de capacidades, reflexividad social significa la capacidad para reflexionar desde la perspectiva e intereses de cada uno de los actores participantes del esfuerzo. Sin este ejercicio, el esfuerzo no será muy diferente de las intervenciones verticales donde prevalecen siempre la visión y las opiniones de los que monopolizan el poder de decidir por monopolizar la gestión de las iniciativas. Bajo esta lógica, compromiso social implica generar conocimiento comprometido con las realidades, necesidades, problemas, desafíos $\mathrm{y}$ aspiraciones de los actores sociales.

\subsection{Control social ampliado de la calidad y relevancia}

En el modo clásico, el control es desarrollado por los pares y es suficiente para determinar la calidad y relevancia del conocimiento generado. En el nuevo modelo de gestión del conocimiento eso ya no es suficiente. La participación de otros actores del contexto implica la incorporación de nuevos criterios para definir lo que es conocimiento relevante. Cada categoría de actor social va a proponer sus criterios para evaluar la calidad del esfuerzo y la relevancia de su impacto. Criterios ambientales, sociales, económicos, políticos, institucionales y éticos serán añadidos a los criterios científicos y técnicos, que obviamente continuarán siendo importantes. Finalmente, es también inevitable la creación de modelos suigeneris de evaluación. Esfuerzos realizados bajo la lógica del nuevo modelo no podrán ser percibidos ni valorizados por modelos diseñados para evaluar esfuerzos realizados bajo la lógica del modo clásico (SOUZA, 2008, 2010).

\section{Consideraciones finales}

En el desarrollo de capacidades en el municipio de Nueva Paz fue preciso tener en cuenta la necesidad de comprender por los actores, que para ello hay que cambiar paradigmas, cambios personales y organiza- cionales, mejora de la calidad de las prácticas y cambios de comportamientos, entre otras exigencias, debieron además comprender que hay que construir y apropiarse de conocimientos significativos del contexto de actuación de los actores para la solución de los problemas; se dan cuenta de la necesidad de un cambio, de despojarse, de borrar de la mente, conocimientos, actitudes y prácticas para la solución de los problemas nuevos y actuales que no pueden ser resueltos con los métodos y técnicas tradicionales;es imprescindible actualizar, transformar e innovar los conocimientos, actitudes y prácticas en la solución de los problemas presentes en el municipio.

\section{Referencias}

ARTIGAS, E. Programa de capacitación para la participación activa de los actores locales en el desarrollo de Nueva Paz. España: Universidad de Málaga, 2014. Disponible en: <http:/ / www.eumed.net/libros gratis/2014/1366/index.htm>.

CENTRO DE ESTUDIOS DE DESARROLLO AGRARIO Y RURAL - CEDAR. Bases conceptuales, filosóficas y metodológicas del Desarrollo Local. La Habana, 2013. p. 20-23.

Programa de Desarrollo Participativo Municipio Inteligente. Habana, 2012.

DEMO, P. Conhecimento moderno: sobre ética e intervenção do conhecimento. Petrópolis, RJ: Vozes, 1997.

DIEZ DEL CORRAL, P. Una nueva mirada a la educación artística desde el paradigma del desarrollo humano. Madrid: Universidad Complutense, 2005.

ENGEL, P.; SALOMÓN, M. Facilitating innovation for development. A RAAKS Resource Box. The Netherlands: STOAS, 1997.

FREIRE, P. Educación y participación comunitaria. En: CASTELLS, M. et al. Nuevas perspectivas críticas en educación. Barcelona: Paidós Educador, 1994

Editores, 2000 Pedagogía de la autonomía. México: Siglo XXI

GIBBONS, M. et al. The new production of knowledge: the dynamics of science and research in contemporary societies. London: SAGE, 1994.

MAX-NEEF, M. A. Desarrollo a escala humana: conceptos, aplicaciones y algunas reflexiones. Barcelona: Nordan Comunidad/Icaria, 1993.

MINISTERIO DE LA MUJER Y DESARROLLO SOCIAL/ MINISTERIO BRITÁNICO PARA EL DESARROLLO INTERNACIONAL - MIMDES - DFID. Lineamientos programáticos para la elaboración de los planes de desarrollo de capacidades. Proyecto Fortalece, 2008

OJEDA, R. Gestión del Conocimiento en el desarrollo local. En: MES. La nueva Universidad Cubana y su contribución a la universalización del conocimiento. La Habana: Editorial Félix Valera, 2007. 
Hoja de ruta hacia un Municipio Inteligente por un Desarrollo Agrario y Rural sostenible. VIII Taller Internacional “Universidad Ciencia y Tecnología. Congreso Internacional de Educación Superior. Febrero 2012. Publicado CD Memorias del Evento: ISBN 978959-1614-34-6., 2012

La Generación de Conocimiento desde la Investigación y la Extensión en el cambio de época para la consecución de municipios inteligentes. XI Taller Internacional: "Comunidades: Historia y Desarrollo". "Lo comunitario en la transformación emancipatoria de la sociedad", 2013.

POZO, A.Mapeo de actores sociales. Lima, Perú, 2007.

RAMOS, A.; OJEDA, R; PEÑA, M.A.; ARTIGAS, E. La Gestión Deportiva Municipal en Iberoamérica: Historia, Teoría y Práctica. El deporte en el ámbito comunitario para el desarrollo humano. España: Editorial Librerías Deportivas Esteban Sanz S. L., 2013.

REBELLATO, J. L. La participación como territorio de contradicciones éticas. En: ROMERO, M. I.; HERNÁN-
DEZ, C. N. Concepción y metodología de la educación popular. Selección de lecturas. La Habana: Editorial Caminos, 2004. Tomo I, p. 299-336.

SOUZA, J. "Changing Roles and Responsibilities of Planners and Planning Units: from rationalization to flexibilization under the networking logic"; forthcoming in Source Book on Planning inAgricultural Research. The Hague, the Netherlands: ISNAR-CAB International, 2001.

SOUZA, J. El Poder de las redes y las redes del poder en el contexto del cambio de época. En: BENALCÁZAR, Patricio Carpio (Editor). Retos del desarrollo local. Quito: DOCUTECH, 2006. p. 81-98.

SOUZA, J. La geopolítica del conocimiento y la gestión de procesos de innovación en la época histórica emergente. Campina Grande, PB: [s.n.], 2008.

SOUZA, J. La pedagogía de la pregunta y el "día después del desarrollo". En: Dirección De Desarrollo Rural. CONGRESO NACIONAL DE EDUCACIÓN RURAL, 3., Medellín, Colombia. Brasil: EMBRAPA, 2010. 